\title{
A Comparison of Voltage Stability Indices
}

\author{
Claudia Reis \\ Polytechnic Institute of Braganca \\ Portugal \\ Email: claudia-reis@ipb.pt
}

\author{
F.P. Maciel Barbosa \\ Faculty of Engineering of Porto University \\ Portugal \\ Email: fmb@fe.up.pt
}

\begin{abstract}
Voltage stability has become a very importante issue of power systems analysis. This paper discusses some important aspects related to voltage stability indices in electric power systems. Some techniques previously studied in the literature are analyzed and a comparison of the performance of several indices is presented. The effectiveness of the analyzed methods are demonstrated through numerical studies in IEEE 14 busbar test system, using several different scenarios of load increase.
\end{abstract}

\section{INTRODUCTION}

Problems related to voltage stability in power systems are one of the major concerns in power system planning and operation.

Voltage stability is concerned with the ability of a power system to maintain acceptable voltages at all nodes in the system under normal condition and after being subject to a disturbance [1].

A power system is said to have a situation of voltage instability when a disturbance causes a progressive and uncontrollable decrease in voltage level.

During the last decades, the voltage stability problem has been given more attention primarily due to a number of stability accidents that occurred in some countries. Some wellknown examples of voltage stability incidents were reported in France, Belgium, Sweden, Germany, Japan and in USA [2], [3].

As power systems become more complex and heavily loaded, along with economical and environmental constraints, voltage instability becomes an increasingly serious problem, leading systems to operate close to their limits. Voltage instability is essentially a local phenomenon, however its consequences may have widespread impact.

The study of voltage stability has been analyzed under different approaches that can be basically classified into dynamic and static analysis.

The static voltage stability methods depend mainly on the steady state model in the analysis, such as power flow model or a linearized dynamic model described by the steady state operation.

The dynamic analysis implies the use of a model characterized by nonlinear differential and algebric equations which include generators dynamics, tap changing transformers, etc, through transient stability simulations [4].

Although stability studies, in general, requires a dynamic model of the power system, in this paper analysis of voltage behaviour has been approached using static techniques, which have been widely used on voltage stability analysis.

An accurate knowledge of how close the actual system's operating point is from the voltage stability limit is crucial to operators. Therefore, to find a voltage stability index has become an important task for many voltage stability studies. These indices provide reliable information about proximity of voltage instability in a power system. Usually, their values changes between 0 (no load) and 1 (voltage collapse).

The voltage stability analysis, using different methods, will be highlighted in this paper and the results obtained from simulating on IEEE 14 busbar test system will be discussed.

\section{INDICES FORMULATION}

The condition of voltage stability in a power system can be known using voltage stability indices. This indices can either reveal the critical bus of a power system or the stability of each line connected between two bus in an interconnected network or evaluate the voltage stability margins of a system. The indices used to examine the system stability are briefly described in this section.

1) P-V and $Q-V$ curves: The P-V curves are the most used method of predicting voltage security. They are used to determine the loading margin of a power system. The power system load is gradually increased and, at each increment, is necessary recompute power flows until the nose of the PV curve is reached. The margin between the voltage collapse point and the current operating point is used as voltage stability criterion [5].

With Q-V curve is possible, for the operators, to know which is the maximum reactive power that can be achieved or added to the weakest bus before reaching minimum voltage limit. The reactive power margin is the MVar distance from the operating point to the bottom of the $\mathrm{Q}-\mathrm{V}$ curve. The $\mathrm{Q}-\mathrm{V}$ curve can be used as an index for voltage instability. The point where $\mathrm{dQ} / \mathrm{dV}$ is zero is the point of voltage stability limit [1].

2) L index: Kessel et al. [6] developed a voltage stability index based on the solution of the power flow equations. The $\mathrm{L}$ index is a quantitative measure for the estimation of the distance of the actual state of the system to the stability limit. The $\mathrm{L}$ index describes the stability of the complete system and is given by: 


$$
L=\max _{j \in \alpha_{L}}\left\{L_{j}\right\}=\max _{j \in \alpha_{L}}\left|1-\frac{\sum_{i \in \alpha_{G}} \underline{F}_{j i} \underline{V}_{i}}{\underline{V}_{j}}\right|
$$

where $\alpha_{L}$ is the set of consumer nodes and $\alpha_{G}$ is the set of generator nodes.

$L_{j}$ is a local indicator that determinates the busbars from where collapse may originate. The $\mathrm{L}$ index varies in a range between 0 (no load) and 1 (voltage collapse).

3) $V / V O$ index: Assuming the bus voltage values (V) to be known from load flow or state estimation studies, new bus voltages (V0) are obtained solving a load flow for the system at an identical state but with all loads set to zero. The ratio V/Vo at each node yields a voltage stability map of the system, allowing for immediate detection of weak and effective countermeasure spots [5].

4) Modal analysis: Gao et al. [7] proposed a method that computes the smallest eigenvalue and associated eigenvectors of the reduced Jacobian matrix of the power system based on the steady state system model. The eigenvalues are associated with a mode of voltage and reactive power variation. If all the eigenvalues are positive, the system is considered to be voltage stable. If one of the eigenvalues is negative, the system is considered to be voltage unstable. A zero eigenvalue of the reduced Jacobian matrix means that the system is on the border of voltage instability. The potential voltage collapse situation of a stable system can be predicted through the evaluation of the minimum positive eigenvalues. The magnitude of each minimum eigenvalue provides a measure to know how close the system is to voltage collapse. By using the bus participation factor, the weakest bus can be determined, which is the greatest contributing factor for a system to reach voltage collapse situation.

5) Line Stability Index $L_{m n}$ : M.Moghavemmi et al. [8] derived a line stability index based on the power transmission concept in a single line, in which discriminant of the voltage quadratic equation is set to be greater or equal than zero to achieve stability. If the discriminant is small than zero, the roots will be imaginary, which means that cause instability in the system. Figure 1 ilustrates a single line of an interconnected network where the $L_{m n}$ is derived from.

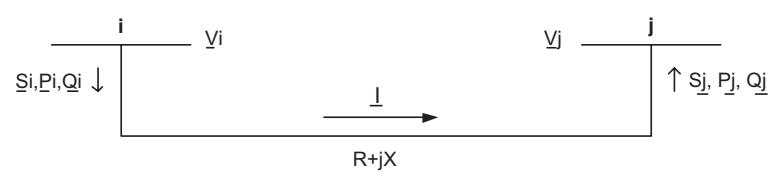

Fig. 1. Typical one-line diagram of transmission line.

The line stability index, for this model, can be defined as:

$$
L_{m n}=\frac{4 X Q_{j}}{\left[V_{i} \operatorname{sen}(\theta-\delta)\right]^{2}}
$$

where $\theta$ is the line impedance angle and $\delta$ is the angle difference between the supply voltage and the receiving end voltage.
Lines that presents values of $L_{m n}$ close to 1 , indicates that those lines are closer to theirs instability points. To maintain a secure condition, the $L_{m n}$ index should be less than 1 .

6) Line Stability Index FVSI: The line stability index FVSI proposed by I.Musirin et al. [9] is based on a concept of power flow through a single line. For a typical transmission line, the stability index is calculated by:

$$
F V S I_{i j}=\frac{4 Z^{2} Q_{j}}{V_{i}^{2} X}
$$

where $\mathrm{Z}$ is the line impedance, $\mathrm{X}$ is the line reactance, $Q_{j}$ is the reactive power flow at the receiving end and $V_{i}$ is the sending end voltage.

The line that gives index value closest to 1 will be the most critical line of the bus and may lead to the whole system instability. The calculated FVSI can also be used to determine the weakest bus on the system. The determination of the weakest bus is based on the maximum load allowed on a load bus. The most vulnerable bus in the system corresponds to the bus with the smallest maximum permissible load.

7) Line Stability Index LQP: The LQP index derived by A.Mohamed et al. [10] is obtained using the same concept as [8], [9], in which the discriminant of the power quadratic equation is set to be greater or equal than zero. The LQP is obtained as follows:

$$
L Q P=4\left(\frac{X}{V_{i}^{2}}\right)\left(\frac{X}{V_{i}^{2}} P_{i}^{2}+Q_{j}\right)
$$

where $\mathrm{X}$ is the line reactance, $Q_{j}$ is the reactive power flow at the receiving bus, $V_{i}$ is the voltage on sending bus and $P_{i}$ is the active power flow at the sending bus.

To maintain a secure condition, the value of LQP index should be maintained less than 1 .

8) Line Stability Indices VCPI: The VCPI indices proposed by M.Moghavvemi et al. [11] investigates the stability of each line of the system and they are based on the concept of maximum power transfered through a line.

$$
\begin{gathered}
V C P I(1)=\frac{P_{R}}{P_{R(\max )}} \\
V C P I(2)=\frac{Q_{R}}{Q_{R(\max )}}
\end{gathered}
$$

where the values of $P_{R}$ e $Q_{R}$ are obtained from conventional power flows calculations, and $P_{R(\max )}$ and $Q_{R(\max )}$ are the maximum active and reactive power that can be transfered through a line.

The VCPI indices varies from 0 (no load condition) to 1 (voltage collapse).

\section{TEST RESULTS AND DISCUSSION}

The voltage stability analysis were performed on IEEE 14 busbar test system. This system has 5 generator busbars, 9 load busbars and 20 interconnected branches. 
The voltage stability margin can be easily calculated with $\mathrm{P}-\mathrm{V}$ curves. This curves show the bus voltage level as the loading factor $\lambda$ increases. The loading factor is 1 at base case and is gradually increased, in all busbars of the system, until maximum loading point is reached, maintaining constant the power factor.

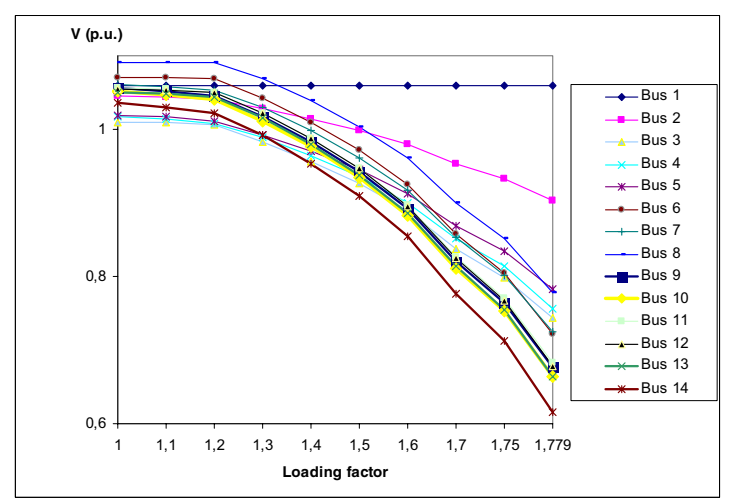

Fig. 2. P-V curves for IEEE 14 busbar test system.

Analysing the Figure 2 we observe that, as the power system load is gradually increased, the voltages at the busbars decreases. As shown in Figure 2, the voltage stability margin of this system is approximated $77,9 \%$.

The Q-V curve calculates the voltage stability margin of a particular bus, i.e., this curves show how much reactive power demand can be increased before the system suffers a voltage collapse.

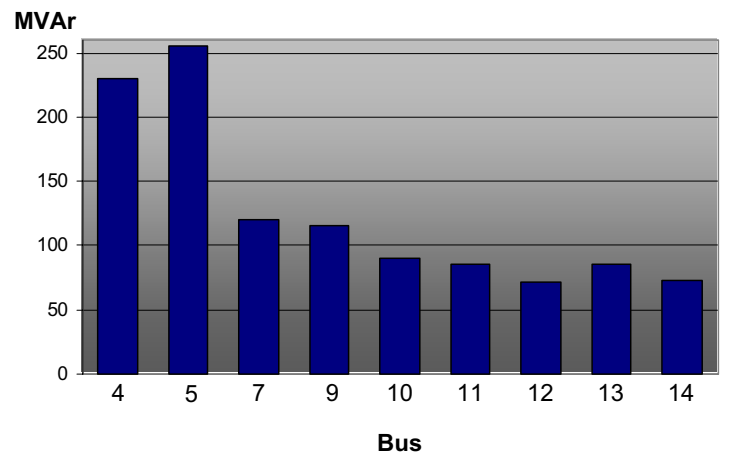

Fig. 3. Margin of reactive power in IEEE 14 bus test system.

In Figure 3, busbars 12 and 14 have the lowest margin of reactive power, which indicates that they are the most critical busbars in the system.

The V/Vo index is very simple to compute. In Figure 4, is possible to observe the index value for two different loading situations: for a condition near the base case (loading factor $\lambda=1,2)$ - situation $\mathbf{A}$ - and for the critical operating case (loading factor $\lambda=1,779$ ) - situation $\mathbf{B}$.

As was expected, in situation $\mathbf{A}$, the system is far away of the voltage instability. This fact can be easily proved because all voltages in the busbars present values very near the ones obtained with the system with all loads a zero (V0). The situation $\mathbf{B}$ reveals that the voltage at the busbars have values very far away of the ones obtained with the system with all loads a zero. So, in situation $\mathbf{B}$, the system is near of the voltage instability. Figure 4 shows that the critical bus of this system is bus 14 , because it presents the smallest index value.

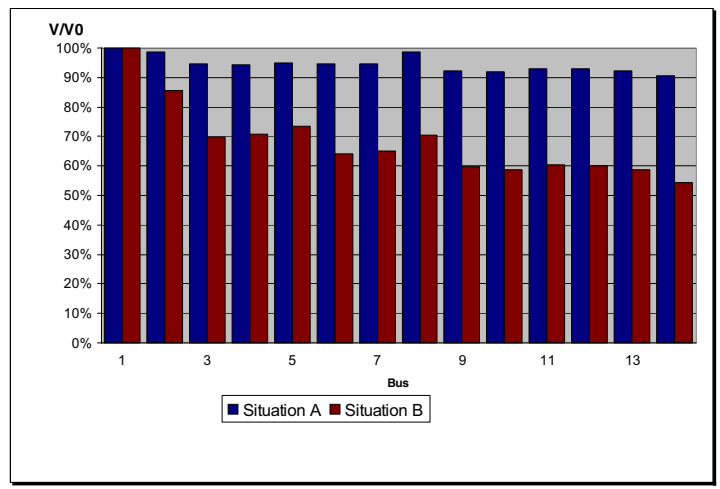

Fig. 4. V/V0 index for IEEE 14 bus test system.

To illustrate the application of modal analysis, we choosed three operating conditions on the P-V curves of Figure 2: the base case, the case of loading factor 1,5 and the critical operating case (loading factor 1,779). All the eigenvalues of reduced reactive Jacobian matrix are positive, indicating that the system is voltage stable at all tested load conditions. The magnitudes of the eigenvalues decrease as the system approaches to instability.

At the critical operating point, the smallest eigenvalue is 1,4369. This value is considered the least stable mode for the critical operating point and is used to determine the bus participation factors. Figure 5 shows the bus participation factors in the least stable mode for the critical operating point.

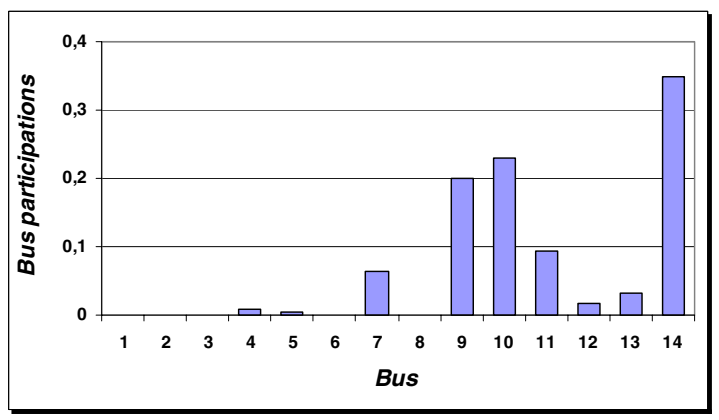

Fig. 5. Bus participation factors in the least stable mode for critical operating case.

It is possible to know, with modal analysis, which bus of the tested system contribute more to the voltage collapse. Figure 5 shows that the critical bus of this system is bus 14 because busbars with high participation factors are considered the most vulnerables. 
The local index $L_{j}$ permits the determination of the weakest bus in the system. Figure 6 shows the values of the local index $L_{j}$ in the IEEE 14 busbar test system.

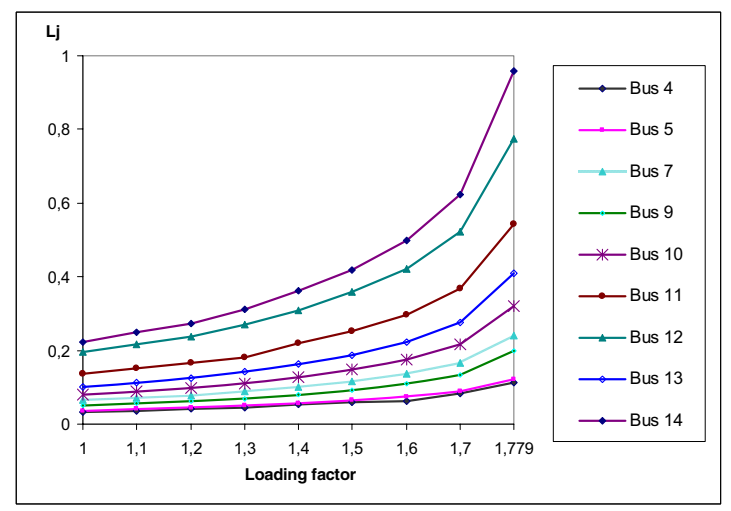

Fig. 6. Evaluation of $\mathrm{Lj}$ index versus load variation.

Figure 6 shows that bus 14 exhibits the highest $L_{j}$ index, which indicates that it is the most vulnerable bus on the system.

In Figure 7, $\mathrm{L}$ index and the voltage at bus 14 (critical bus) are plotted as a function of loading factor.

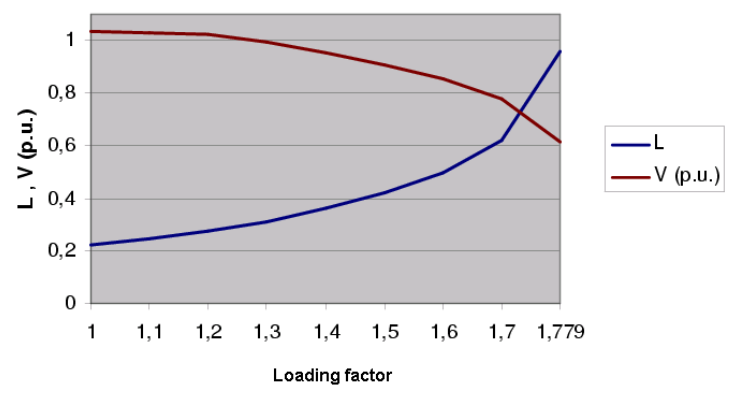

Fig. 7. Stability indicator $\mathrm{L}$ and its relation to the critical voltage.

In the critical operating point $\mathrm{L}=0,958$, so the voltage stability of this system is guaranteed. The stability limit is reached for $\mathrm{L}=1$.

To investigate the effectiveness of the line stability indices, the reactive load were gradually increased, only in one bus at a time, from the base case until its maximum allowable load, keeping the load at the other busbars fixed at base load. The test was carried out for several buses but only the cases of buses 10, 11 and 14 are presented. Table 1 shows the stressed conditions of the lines for the maximum loadability of the buses. The values of the line stability indices are maximum at the maximum loadability of each individual load bus. The line that presents the largest index with respect to a bus is considered the most critical line of that bus. From Table 1 it is observed that the line that connect bus 9 to bus 10 is the most critical line referred to bus 10 , the line 6-11 is the most critical line with respect to bus 11 and line 9-14 is the most critical line of bus 14 .
This table also illustrates that, for any state of loading, $\operatorname{VCPI}(1)$ is always equal to $\operatorname{VCPI}(2)$.

Table 1 shows that the performance of the line indices studied has high degree of accuracy, reliability and the results are very closed in agreement. Line stability indices can also determine the weakest bus in the system and it is based on the maximum permissible load. It is observed that buses 10,11 and 14 indicates 0,948 p.u., 0,855 p.u. and 0,728 p.u. as the maximum permissible reactive load, respectively. Since bus 14 has the smallest maximum loadability, it is considered the most critical bus.

\begin{tabular}{|c||c|c|c|c|c|c|}
\hline \multicolumn{7}{|c|}{ Table 1 - Line stability indices for reactive power load } \\
\hline Load (p.u.) & Line & Lmn & FVSI & LQP & VCPI(1) & VCPI(2) \\
\hline Q10=0,948 & $9-10$ & 0,5238 & 0,5405 & 0,4741 & 0,495 & 0,495 \\
\hline & $10-11$ & 0,5213 & 0,5346 & 0,4557 & 0,4947 & 0,4947 \\
\hline \hline Q11=0,855 & $6-11$ & 0,7723 & 0,8272 & 0,681 & 0,7311 & 0,7311 \\
\hline & $10-11$ & 0,6265 & 0,6673 & 0,5692 & 0,5969 & 0,5969 \\
\hline \hline Q14=0,728 & $9-14$ & 0,8475 & 0,8994 & 0,756 & 0,8184 & 0,8184 \\
\hline & $13-14$ & 0,801 & 0,8493 & 0,7 & 0,7668 & 0,7668 \\
\hline
\end{tabular}

\section{CONCLUSION}

This paper presents a comparative study and analysis of the performance of some online static voltage collapse indices. The application of those indices on IEEE 14 bus testing system gave accurate results. The shown simulations indicate that the bus 14 of IEEE 14 busbar test system is considered the weakiest bus in the system. Line indices provide an accurate information with regard to the stability condition of the lines. The research shows an agreement between the different line stability indices.

\section{REFERENCES}

[1] P.Kundur "Power System Stability and Control" McGraw-Hill, New York, 1994

[2] "Voltage Stability of Power Systems: Concepts, Analytical Tools and Industry Experience", IEEE Committee Vol.IEEE/PES 93TH0358-2PWR 1990.

[3] K. Takahashi and Y. Nomura "The Power System Failure on July 23rd 1987 in Tokyo" CIGRE SC-37 Meeting 37.87(JP)07(E) 1987.

[4] J.C. Chow, R. Fischl and H. Yan "On the Evaluation of Voltage Collapse Criteria” IEEE Trans., PWRS-5, pp. 612-620, May 1990

[5] Editor/Coordinator: Claudio Canizares "Voltage Stability Assessment: Concepts, Practices and Tools" IEEE/PES Power System Stability Subcommittee Special Publication, August 2002

[6] P.Kessel, H.Glavitsch "Estimating the Voltage Stability of a Power System" IEEE, Transactions on Power Delivery, Vol.PWRD-1, N3, July 1986

[7] B.Gao, G.K.Morison, P.Kundur "Voltage Stability Evaluation Using Modal Analysis" IEEE, Transactions on Power Systems, Vol.7, N4, November 1992

[8] M.Moghavvemi, F.M.Omar "Technique for Contingency Monitoring and Voltage Collapse Prediction" IEEE Proceeding on Generation, Transmission and Distribution, Vol. 145, N6, pp. 634-640 November 1998

[9] I.Musirin, T.K.A.Rahman "Novel Fast Voltage Stability Index (FVSI) for Voltage Stability Analysis in Power Transmission System" 2002 Student Conference on Research and Development Proceedings, Shah Alam, Malasia, July 2002

[10] A.Mohamed, G.B.Jasmon, S.Yusoff "A Static Voltage Collapse Indicator using Line Stability Factors" Journal of Industrial Technology, Vol.7, N1, pp. 73-85, 1989

[11] M.Moghavvemi, O.Faruque "Real-Time Contingency Evaluation and Ranking Technique" IEEE Proceeding on Generation, Transmission and Distribution, Vol. 145, N5, September 1998 